\title{
Research on System of Marine Situation Information Analysis and Early Warning based on Artifical Intelligence
}

\author{
Long Ziye ${ }^{1, a}$, Wang Peng ${ }^{2, b}$, Lin Zhiwen ${ }^{1, c}$ and Zhu Jing ${ }^{1, d}$ \\ 1'Department of Navy Research Academy, Beijing102249 \\ 2Institute of Computing Technology of Chinese Academy of Science, Beijing 100080 \\ alongziye@tom.com, bwangp@ict.ac.cn, Ilzwdzs@tom.com, ${ }^{\mathrm{d}}$ zhujing1116@126.com
}

\begin{abstract}
Keywords: Artificial Intelligence, Situation Intelligence Analysis, Internet of Things;
Abstract. With the rapid development of artificial intelligence and the development of intelligent hardware and equipment, the data has gradually changed from a single text mode to a diversified data. Diversified data is not only large in size but also diverse in scope, so it is not easy to conduct statistical analysis. With the development of artificial intelligence and the Internet of Things, these challenges will be solved. To address these issues, carry out studies on the system of maritime situational intelligence analysis and early warning. The design of marine situation intelligence analysis and early warning system based on artificial intelligence is proposed and the key technologies are analyzed. The application of artificial intelligence technology in maritime situational intelligence analysis and early warning not only can improve the efficiency of civil actions such as rescue at sea, disaster relief, but also can greatly enhance maritime military competitiveness and ensure the safety of maritime areas.
\end{abstract}

\section{Introduction}

With the development of big data and artificial intelligence, data has become an important resource. The development of the Internet of Things realizes the connection of objects, and the information carried by the sensors is also an important data resource. In particular, in the system of maritime situational intelligence analysis and early warning, it is inseparable from the data carried by the sensors. On the one hand, with the rapid development of information technology, we can easily access a large amount of information, resulting in the phenomenon of information overload. On the other hand, the information we obtain is encompassing, with complicated contents, mixed actual and real situations, phenomenon. How to get effective information from these overload information has become an urgent problem to be solved. How to mine and extract accurate and valuable intelligence information from these overloaded data has become an urgent problem to be solved[1]. After processing valuable data can be described as information assets, not the traditional sense of the data, such as shipping a large number of loose bulk shipping Matsai shipping data collected after the formation of multidimensional big data base model, which use the processing of big data and show the way, using big data methods and thinking, through the big data services and innovative products to enhance the efficiency of shipping services. Visible big data value is reflected in all aspects of life in the industry. How to find the potential value of the processed big data itself is a challenge.Based on the Internet of Things Situation Intelligence Big Data, this paper applies the methods of big data, Internet of Things and artificial intelligence to carry out the research and design of the system of analysis and early warning of the situation information on the sea, and puts forward the systrm of marine situation analysis and early warning based on artificial intelligence,and related core technologies were analyzed.

\section{Research Background}

Situational Awareness Concept. All of us now live in a man-machine-environment (natural and social) system with an increasingly active information. The command and control system is naturally through the human-computer interaction between the environment and the input of information, 
processing, output, feedback to adjust the ongoing theme of activities, thus reducing or eliminating the uncertainty of the outcome of the process. At the heart of command and control systems, Micha R. Endsley presented a consensus concept on Situation Awareness (SA) at the 1988 Human Factor Annual Meeting, "the perception of the elements in the environment within a volume of time and space, the comprehension of their meaning, and the projection of their status in the near future." That is, within a certain time and space perception of the various components of the environment, understanding, and then predict the subsequent changes in these components.

Situation awareness is the traditional equipment and intelligent devices for integrated networking, and combined with some new programs such as big data to generate a situational awareness platform. Situational Awareness gained the information from each sensor is combined with big data to bring valuable intelligence to the visualization platform. The basic principle of situation-aware data acquisition in this process is that the traditional ips, firewall, Trojan analysis and the dynamic detection the future of everyone is proud of that are all the sources of situation-aware data acquisition. After the data is acquired, how to process the data, The data should be what kind of ability to be easy to use, these are uncertain need to be analyzed, the use of artificial intelligence technology analysis is now the mainstream method, but also the focus of this paper.

The Concept of LoRa. Situational awareness is the main way to obtain information on maritime situational awareness. The maritime situational awareness platform must rely on the Internet to form a cognitive platform. Due to the complex marine environment, the network is relatively unstable, so a stable network is also the key. LoRa can solve this problem very well.

LoRa is a radio frequency spread spectrum modulation and demodulation technology that is compatible with other modulation schemes such as FSK (Frequency Shift Keying), GMSK (Gaussian Minimum Shift Keying), BPSK (Binary Phase Shift Keying) program formed a clear contrast. It combines digital spread spectrum, digital signal processing and forward error correction coding technology, with unprecedented performance. Previously, only a few military communications systems would incorporate these technologies, and with the introduction of LoRa, the situation in embedded wireless communications has changed radically. LoRa modems are configured to divide the range from 64-4096 chips / bit. AngelBlocks configures the modem to use the highest spreading factor of 4096 chips / bits (12). In contrast, ZigBee can only be divided into the range of 10-12 chips / bit. Through the use of high spreading factors, LoRa technology allows small-capacity data to be transmitted over a wide range of radio spectrum. In fact, when you measure with a spectrum analyzer, the data looks like noise, but the difference is that the noise is irrelevant and the data is correlated, so the data can actually be extracted from the noise.

Therefore, the use of LoRa technology to obtain a wider transmission range and distance at low transmit power is well suited to the complex situation of large sea coverage, which is exactly what we need.

Research on Marine Situation Information Analysis and Early Warning System. The maritime situation intelligence needs to grasp the relevant information of various systems such as the Sentinel system, the navigation system, the operation system and the duty system. The types of the information are important and complicated. The sources of intelligence data include not only early warning reconnaissance information collected by various sensors and reconnaissance but also basic environmental information as well as publicly available open source intelligence information covering data types such as telegrams, images, videos, and altitudes. After data preprocessing such as data screening, conversion and identification, intelligence information of command decision and situational awareness is obtained. After the intelligence information is integrated, analyzed and 
judged according to the tasks and so on, the target intelligence, dynamic intelligence and special intelligence are generated according to the information security requirements And other types of products, and distributed to information users according to the subscription relationship ${ }^{[2]}$, so the analysis of maritime situational intelligence is crucial. The emergence of diversified and complex intelligences has posed many challenges to maritime situational intelligence. The following are some of the major challenges:

The diversity and complexity of intelligence. Now there are many ways to obtain data, which presented in various forms include of structured unstructured data, the form of complex analysis.

Intelligence analysis more difficult. Increasing the complexity of intelligence leads to an increase in the difficulty of analysis.

The complicated maritime environment makes it difficult to deploy the situation awareness platform.

\section{Desing of intelligence analysis and early warning system architecture based on AI}

This section analyzes and designs the system architecture of intelligence analysis and early warning, and introduces it in detail.

The Overall System Architecture Design. The overall system architecture diagram shown in Figure 1, the overall system architecture is divided into two parts: intelligence situational awareness platform, intelligence platform for early warning.

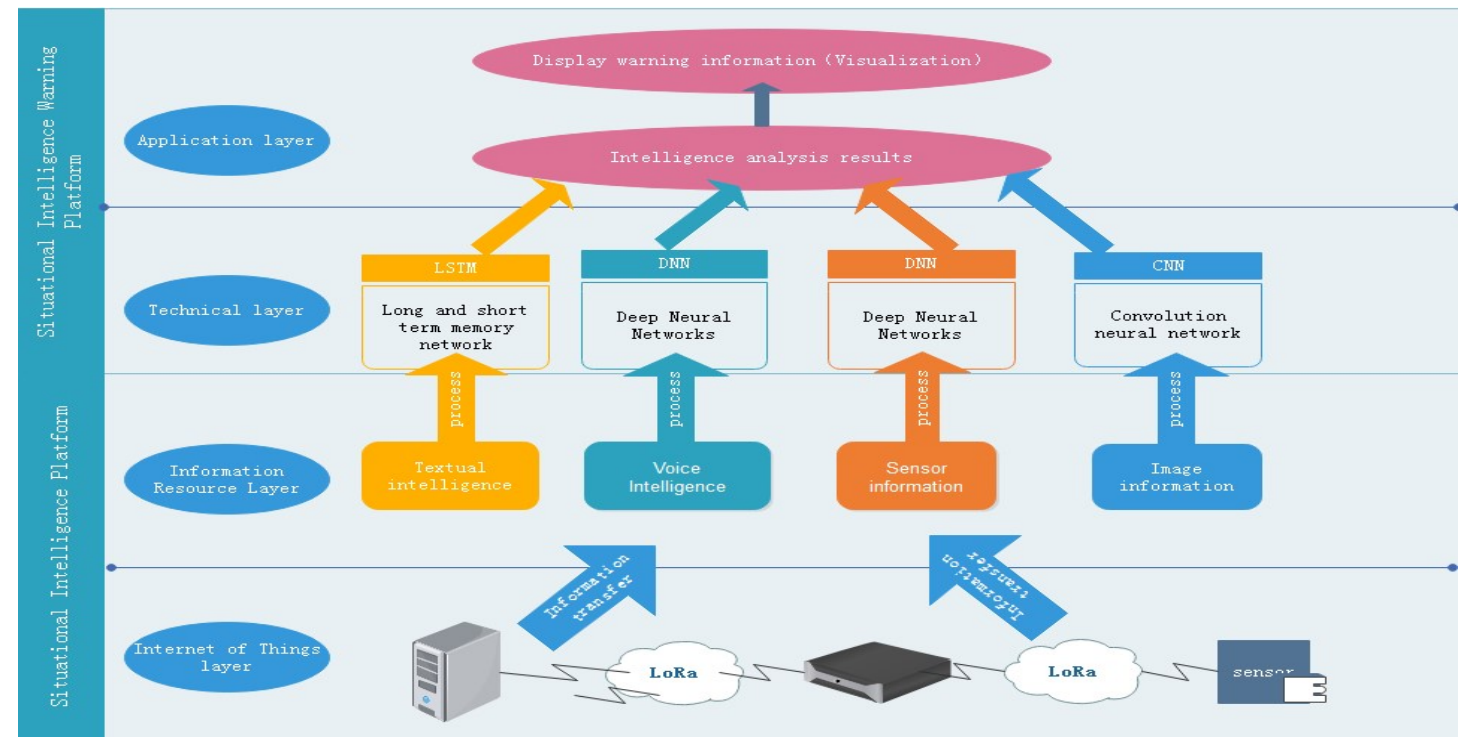

Figure 1: system architecture

Intelligence situational awareness platform. The platform is the source of situational intelligence and a platform for obtaining information. There are two parts of IoT layer and information resource layer.The IoT layer is the bottom layer of the overall architecture and provides a physical support environment for the system to run. The IoT layer provides the basic hardware resources required for computing, storage, networking and other basic software resources such as operating systems and application software for system construction and operation, and provides resources scheduling management, configuration management and status monitoring[3], for the information resource layer to store and transmit data to provide support. Information Resource Layer: It is a part of data resources that integrates all kinds of data resources in the system to provide a unified data resource collection, integration and logical description capabilities. It mainly consists of file intelligence, voice intelligence, sensor information and image information. After simple preconditioning, it is transformed into a format of data and intelligence acceptable to the technical 
layer in the platform of intelligence situation early warning, and provides data intelligence support for the technical layer.

Intelligence platform for early warning. The platform is to deal with the analysis of data intelligence and make early warning judgments platform, the main technical and application layers of two parts. The technology layer uses artificial intelligence-related technologies such as text intelligence applications by receiving data intelligence at the information resource layer, processes the text in the LSTM (long and short term memory networks) for processing the text and compares the thresholds with language models in the natural language processing technology output; sensor information through DNN (deep neural network) processing the sensor information into vector information for analysis and processing of output results. The technical layer is the application of artificial intelligence technology to analyze maritime situational intelligence, and determine whether the warning. The application layer relies on the output of the technology layer to apply the visualization technology, and the output result is displayed to the user through various forms.

The environmental situation in the whole space is displayed, and the marine environment is visualized in the best way.

Operating Mode. Based on the operation mode of big data-based intelligence analysis and service system, according to the needs of the maritime situation, the system dynamically introduces a variety of sources and types of intelligence data, including early warning radar and sensors and other sources as well as texts, graphic images and videos, voice and other carrier types. According to the information security requirements, the intelligence obtained from the system analysis is automatically pushed to the joint command post, regional command post, intelligence users and other authorized users according to the user's subscription/distribution relationship. According to the principle of distributed organization management and resource sharing, the system uses distributed operation management technology to carry out unified management and control of intelligence analysis tasks, calculation capabilities and data resources, and achieve coordinated dispatching based on the information security requirements to complete the task of intelligence analysis. Service-oriented architecture is adopted to systematically encapsulate the core information analysis software including image intelligence analysis, text intelligence analysis, speech intelligence analysis, sensor intelligence analysis and early warning radar data services. The system also relies on the service registration mechanism Unified classification management, the formation of software service resources directory, to achieve intelligence analysis functions in the system of sharing between nodes ${ }^{[3]}$.

\section{Key Technical analysis}

Artificial Intelligence-based Marine Situation Information Analysis and Early Warning System mainly includes image recognition based on convolutional neural network $(\mathrm{CNN})$, natural language processing based on LSTM, deep neural network (DNN) Voice separation technology. These technologies collectively referred to as neural network technology, originated in the fifties and sixties of last century, when the machine was called perception, with input layer, hidden layer and output layer. The input eigenvector reaches the output layer through the hidden layer transformation, and the classification result is obtained at the output layer. The single-layer perceptron has a big problem that can not solve the nonlinear problem such as XOR. It was not overcome by multilayer perceptron invented by Rumelhart et al until the eighties of last century. CNN, LSTM, DNN are based on the improvement of multi-layer perceptron machine. Figure 2 is a multi-layer perceptron machine structure. The implications of multi-layer perceptrons are that the number of layers in a neural network directly determines its ability to portray reality-using fewer neurons per layer to fit more complex functions ${ }^{[4]}$. 


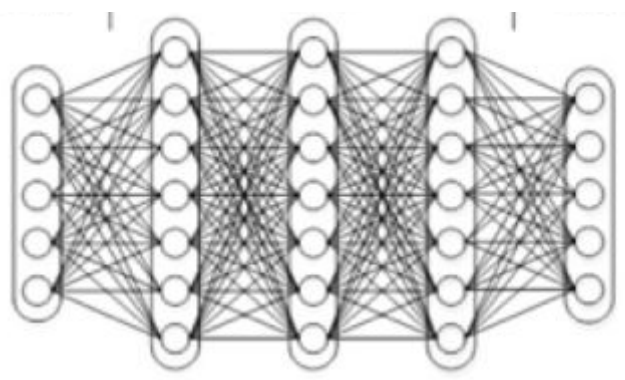

Figure 2: Upper and lower neurons are all connected to the neural network- multi-layer perceptron

Speech separation technology based on deep neural network (DNN). The connection between traditional BP neural network and different layers of neurons in DNN (Deep Neural Network) is "fully connected", that is, the input of one neuron in this sub-layer will accept the last output of each neuron, which way is "fully connected neural network." The goal of speech separation is to separate the target speech from the background noise. In signal processing, speech separation is a very basic type of task and is used in a wide variety of applications including hearing implants, mobile communications, robust automated speech, and speaker recognition. The human auditory system can easily separate one's voice from another ${ }^{[5]}$.

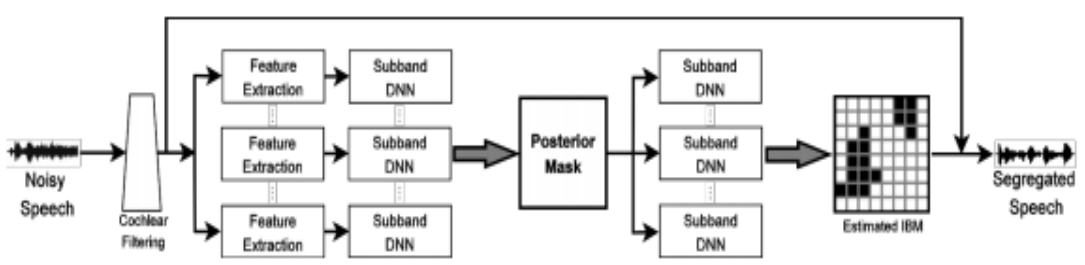

Figure 3: Illustration of second-order DNN for speech separation ${ }^{[6]}$

Figure 3 is a two-tier DNN icon in speech separation, the predecessor has done experiments and achieved good results. Can be seen, DNN in voice separation has been very good application.

Image Recognition Technology Based on Convolutional Neural Network (CNN). Fully connected neural networks because of the large number of parameters such as weight and bias, resulting in very slow convergence of training. In particular, training data of millions of pixels, such as images, can theoretically converge, but it will take at least $\mathrm{N}$ years to produce results and its generalization will be worsened. Convolutional neural network CNN is born to solve this problem, its core features are three:

Locality: For an image, we do not actually need each neuron to accept the entire picture of the information, but to different regions of the neurons corresponding to a different part of a picture, and finally only need to relocal information into together on it. This is equivalent to a dimensionality reduction in the initial input layer of the neuron.

Convolutional weight-sharing: This can be said that convolutional neural network is the most important part of the fully connected neural network is the weight of the largest problem too many parameters, and the convolution of convolutional neural network, different neurons weight is shared, which makes the parameters of the entire neural network greatly reduced, improving the training performance of the entire network.

Pooling Layer: Simple understanding can be regarded as a "lossy compression" of the image, because in actual training, we do not need to extract and train every detail in the image, so the role of pooling is further information abstraction and feature extraction, of course, it will reduce the amount of data processing. One of the reasons that the most widely used application scene of convolutional neural 
networks is image recognition is that the weight sharing makes the network greatly reduce the number of parameters when processing images and make the training speed of the network faster.

Natural Language Processing Technology Based on LSTM (LSTM). LSTM can be used to process timing data and is widely used in fields such as natural language processing and speech recognition. Compared with the original recurrent neural network RNN, LSTM solves the problem of gradient disappearance of RNN and can process long sequence data, becoming the most popular RNN variant at present. Suppose our model input is followed by the input of a word for each word, we need to do word classification, for example, there are two sentences (1) arrive Beijing on November 2nd, where Beijing is the destination; (2) leave Beijing on November 2nd, where Beijing is the starting point. If the input is 'Beijing' using a normal neural network, then the output must be certain, but in fact we would like 'Beijing' to be identified as the destination in front of 'Beijing' when it is 'arrive' in front of 'Beijing' When 'leave', 'Beijing' is recognized as a departure place. Here LSTM will come in handy, because LSTM can remember the historical information, read 'Beijing', LSTM also know in front of 'arrive' or 'leave', according to the historical information to make different judgments, even if the input is the same The output will be different.

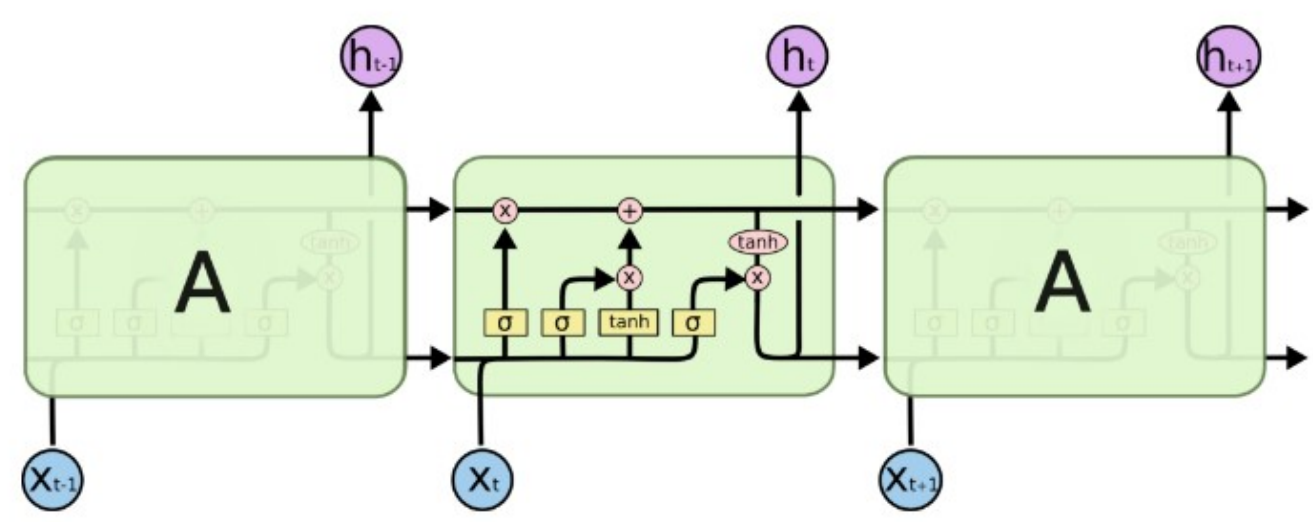

Figure 4: LSTM internal structure

LSTM internal structure from left to right in turn by is composed of the forgotten door, input door, the output door, the three door signals are in real numbers between 0 to 1,1 on behalf of fully open, 0 on behalf of the closure. Forgotten door: Determines whether the memory in the previous moment is remembered or not. When the forgotten door is opened, the previous moment's memory is reserved. When the forgotten door is closed, the previous moment's memory is emptied. Input Gate: Determines how much of the current input is preserved, because not all of the information entered at each time in the sequence input is equally important. When the input is completely useless, the input gate is closed, which is the input at this moment The information was discarded. Output Gate: Determine how much of the current memroy information will be output immediately. When the output gate is opened, it will be all output. When the output gate is closed, the information in the current memory will not be output.

\section{Conclusions}

With the development of big data, Internet of Things and artificial intelligence, the intelligence analysis of maritime situational intelligence based on artificial intelligence has become a new solution to the problem. Based on the artificial intelligence technology, advanced solutions and systems are constructed. Based on the artificial intelligence technology, this paper puts forward a system of marine intelligence analysis and early warning based on artificial intelligence, studies and analyzes it, and analyzes the key technologies therein. In the era of big data artificial intelligence Internet of 
Things, artificial intelligence-based analysis and warning of maritime situational intelligence has become a trend, which is of great significance to civil and military activities at sea.

\section{References}

[1] Hui Chaoyang, Blues. Based on the analysis of military intelligence [J]. Modern Intelligence, 2009,29 (11): 43-45

[2] Jiang Panlin. Big Data Universal Processing Platform and Its Potential Military Applications in ISR [J]. Communication Confrontation, 2013,32 (3): 1-5.

[3] Guo Jiguang, Huang Sheng. Research on military intelligence analysis and service system architecture based on big data [J]. Journal of China Academy of Electronic Sciences, 2017,12 (4): 389-393

[4] Bengio Y. Learing Deep Architectures for AI [J]. Foundations \& Trends in Maching Learing, 2009, 2 (1): 1-127

[5] Wang, DeLiang and Jitong Chen. "Supervised Speech Separation Based on Deep Learning: An Overview." CoRR abs / 1708.07524 (2017): n.pag.

[6] EW Healy, SE Yoho, Y. Wang, and DL Wang, "An algorithm to improve speech recognition in noise for hearing affected pilots," J. Acoust. Soc. Am., Vol. 134, pp. 3029-3038, 2013. 